\title{
Material
}

\section{Self-Care among Adolescents with Type 1 Diabetes Mellitus and Nursing Care in Sri Lanka: Review of Literature}

\author{
A.M.S. Deepanie Pathiranage ${ }^{1}$, Yoshie Mori ${ }^{2}$ and Shamya De Silva ${ }^{3}$ \\ 1 Department of Nursing, Faculty of Allied Health Sciences, University of Peradeniya, Augusta Hill, Peradeniya, Sri Lanka \\ 2 Gunma University Graduate School of Health Sciences, 3-39-22, Showa-machi, Maebashi, Gunma 371-8514, Japan \\ 3 Department of Pediatrics, Faculty of Medicine, University of Colombo, Kynsey Road, Colombo, Sri Lanka
}

\begin{abstract}
Type 1 Diabetes Mellitus (T1DM) is a chronic condition with long term psychological and behavioural implications. Self-care is the cornerstone to adapt into this condition. Sociocultural appropriate self-care practices are vital aspects in nursing care. The article reviews literature on self-care among adolescents with T1DM considering sociocultural context and to discuss existing nursing care and its current issues within the Sri Lankan context. A systematic literature survey was conducted to review empirical studies from 2000 to 2014 . Electronic databases PubMed and CINAHL were utilized. Personal interviews and relevant websites were accessed to explore existing nursing care and its current issues in Sri Lanka. PubMed displayed 1,065 articles for the key words 'nursing' and 'Type 1 diabetes' while CINAHL displayed 31 articles for the same. 'Perceptions', 'associated characteristics', 'school behaviour' and 'family behaviour' were identified as main categories in the literature. The nursing oriented articles relevant to self-care', 'sociocultural', 'Sri Lanka' and 'Type 1 diabetes' were insufficient in number. Scientific studies pertaining to the field need to optimized. The unavailability of specialized nurse educators for childhood diabetes is a noticeable issue. The implementation of effective nursing interventions considering Sri Lankan sociocultural context is recommended.
\end{abstract}

\section{Article Information}

\begin{tabular}{l} 
Key words: \\
Type 1 diabetes, \\
adolescents, \\
self-care, \\
nursing, \\
sociocultural \\
\hline Publication history: \\
Received: November 25, 2015 \\
Revised: December 7, 2015 \\
Accepted: December 10, 2015 \\
Corresponding author: \\
Yoshie Mori \\
Gunma University Graduate School of Health Sciences, \\
3-39-22, Showa-machi, Maebashi, Gunma 371-8514, Japan \\
Tel: +81-27-220-8924 \\
E-mail: myoshie @ gunma-u.ac.jp
\end{tabular}

\section{Introduction}

Diabetes Mellitus is a common metabolic disease in children. The majority of children with diabetes have Insulin Dependent Diabetes Mellitus (hereinafter IDDM). This condition is known as Type 1 Diabetes Mellitus (hereinafter T1DM). It is caused by insulin deficiency usually following autoimmune destruction of the insulin-producing pancreatic beta cells. ${ }^{1}$ T1DM is a psychological and behavioural demanding chronic condition. ${ }^{2}$

The incidence of T1DM has increased steadily among children and adolescents worldwide over past few years. The incidence increases with age and is high among children between 10-14 years of age. ${ }^{3}$ The incidence of T1DM in Sri Lanka has not been identified due to unavailable of accurate, collective data recording of children and adolescents with T1DM. But the incidence has been increasing over the past few years in Sri Lanka. ${ }^{4}$ The accurate epidemiological data of T1DM is not available in Sri Lanka. But clinical evidence and experience based unverified estimates show prevalence of T1DM is between $1-2 \%{ }^{5}$

Although T1DM is not a common in South East Asian (hereinafter SEA) countries, rising prevalence of this condition is a trend. The government and policy makers largely ignored this condition as only a few people being affecting this condition, but huge emotional burden, social stigma, economic problems have mostly affected to their lives among T1DM in SEA unlike the western world. ${ }^{6}$ There are challenging issues 
in management of T1DM in SEA countries. Some population groups in SEA follow non scientific home ground remedies to treat diabetes. Some people did not understand implications of disease management due to lack of awareness and low literacy rates. Misconception affects on nutrition management. Some restricts the exercise due to fear of hypoglycemia. The social stigma attached to the T1DM patients direct them to not to disclose the condition. ${ }^{5}$ Reveal of scientific facts in this area in Sri Lanka is vital to promote effective nursing care for the patients with T1DM, especially self-care practices among adolescents with T1DM.

This article aims to review existing literature on self-care among adolescents considering socio cultural context and to explore existing nursing care and current issues of them for discussing desirable nursing intervention in Sri Lanka.

\section{Methods}

A literature search was conducted using electronic databases PubMed and Cumulative Index to Nursing and Allied Health Literature (CINAHL) from the site of Hinari Research in Health. Hinari access to Research in Health Programme provides access to large collection of biomedical and health literature. The empirical studies from 2000 to 2014 were focused on considering self-care in adolescents with T1DM. The systematic review was done using the key words 'diabetes', 'Type 1 diabetes' and 'adolescents', 'selfcare', 'self management', 'nursing', 'sociocultural' and 'Sri Lanka' together with 'Type 1 diabetes'. Because of lack in nursing oriented empirical studies in this field in Sri Lanka, internet websites and personal interviews were used additionally to explore existing nursing care for the adolescents with T1DM and current issues of them in Sri Lanka.

\section{Results}

The number of articles were reviewed in databases of PubMed/Hinari and CINAHL/Hinari from January 2000 to December 2014. A considerable number of articles resulted in using the key words 'diabetes' and 'Type 1 diabetes'. PubMed and CINAHL displayed 338,315 and 36,154 articles respectively for the key word 'diabetes'. The key word 'Type 1 diabetes' resulted in 38,778 articles in PubMed and 3,811 articles in CINAHL. Browsing articles using the key words 'Type 1 diabetes' and 'Adolescents' resulted in 9,239 and 456 articles respectively in PubMed and CINAHL. 'Type 1 diabetes' and 'self-care or self management' resulted in 2,882 PubMed articles and 197 articles in CINAHL. PubMed displayed 1,065 articles for the key words 'nursing' together with 'Type 1 diabetes' while CINAHL displayed 31 articles for the same.

'Type 1 diabetes' and 'adolescents' and 'self-care' or 'self management' resulted in 1,266 articles in PubMed, whereas CINAHL displayed 69 articles. 'Type 1 diabetes' and 'adolescents' and 'self-care' or 'self management' and 'nursing' resulted in 164 articles in PubMed and 5 articles in CINAHL.

Ten articles from PubMed and one article from CINAHL resulted for the key words 'Type 1 diabetes' and 'Sri Lanka'. Only three PubMed articles and one CINAHL article displayed for the key words 'sociocultural' together with 'Type 1 diabetes'. The 'diabetes' and 'nursing' related articles in relation to 'Sri Lanka' (4 PubMed and 1 CINAHL) and 'Sri Lankan sociocultural' context (0 PubMed and 0 CINAHL) were limited.

The figures 1 and 2 show the pattern which presented as number of articles in each year the data base 'PubMed' and figures 3 and 4 represented CINAHL articles. Same patterns of results could be identified in both databases. The figures show how the numbers of articles increase since 2000 to 2014. The figures 1 and 2 clearly show that the increment of 'Type 1 diabetes' related articles is lower than the 'diabetes' related articles in both databases. In contrast, yearly increment articles are in lower level for the key words 'nursing,, 'socio cultural' and Sri Lanka together with 'Type 1 diabetes' rather than the key words 'adolescents' and 'self care' or 'self management' (Figs. 3 and 4).

Almost all the articles among ten, related to 'Type 1 diabetes' and 'Sri Lanka' were more medical oriented studies. Only one article was a study on young diabetes and two articles were updates of diabetes in SEA countries. The influence of culture on self-care management was described in detail in articles related to Type 2 diabetes. Two ethnographic studies were identified in the articles related to Type 2 diabetes. None of the articles have described self-care behavior among children and adolescents within the Sri Lankan sociocultural context.

The resulted articles for combination of key words 'Type 1 diabetes' and 'nursing' were utilized to analyze the content of the articles. The total number of articles (31) resulted in CINAHL and randomly selected 92 articles in PubMed were accessed to identify main categories which relevant to sociocultural nature. Fifty-two articles has encountered in this regard.

Four main categories were identified by the careful evaluation of the literature, (a) perception; (b) associate characteristics; (c) school behaviour; (d) family behaviour. Most articles related to 'perception' mainly referred the perceptions of children, ${ }^{7,8}$ perceptions of mothers. ${ }^{9,10}$ Some articles described perceptions of school nurses. ${ }^{11}$

Ethnicity, age, gender, ${ }^{12,13}$ authoritative parenting, ${ }^{14}$ different cultural and ethnic groups,${ }^{15}$ maternal seperaration anxiety, ${ }^{16}$ characteristics of child, nature of the illnesses, support from parents, school environment, health care team, ${ }^{17}$ socio economic status, family parenting style, extra familial network, personality, beliefs, knowledge, self efficacy and skills and coping behaviour ${ }^{13}$ were described in ralation to 'associated characteristics'. 


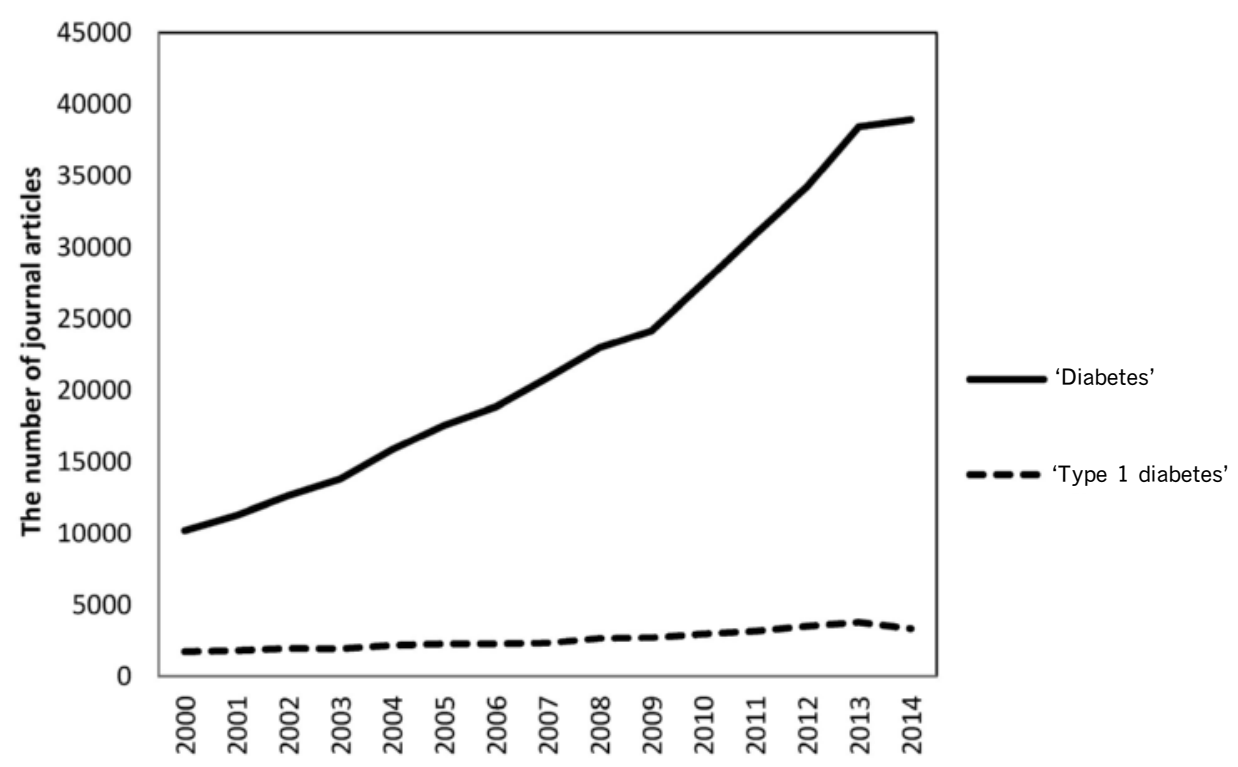

Fig. 1 Number of journal articles found in PubMed/Hinari 2000-2014 for the key words: 'diabetes' and 'Type 1 diabetes'.

Criteria -PUB MED/HINARI

Time: Jan YEAR- Dec YEAR

Publication Type: Journal articles

Language: English

Key word search in Title / Abstract
Criteria- CINHAL / HINARI

Time: Jan YEAR -Dec YEAR publication type: Journal articles Language: English

Key word search in Abstract

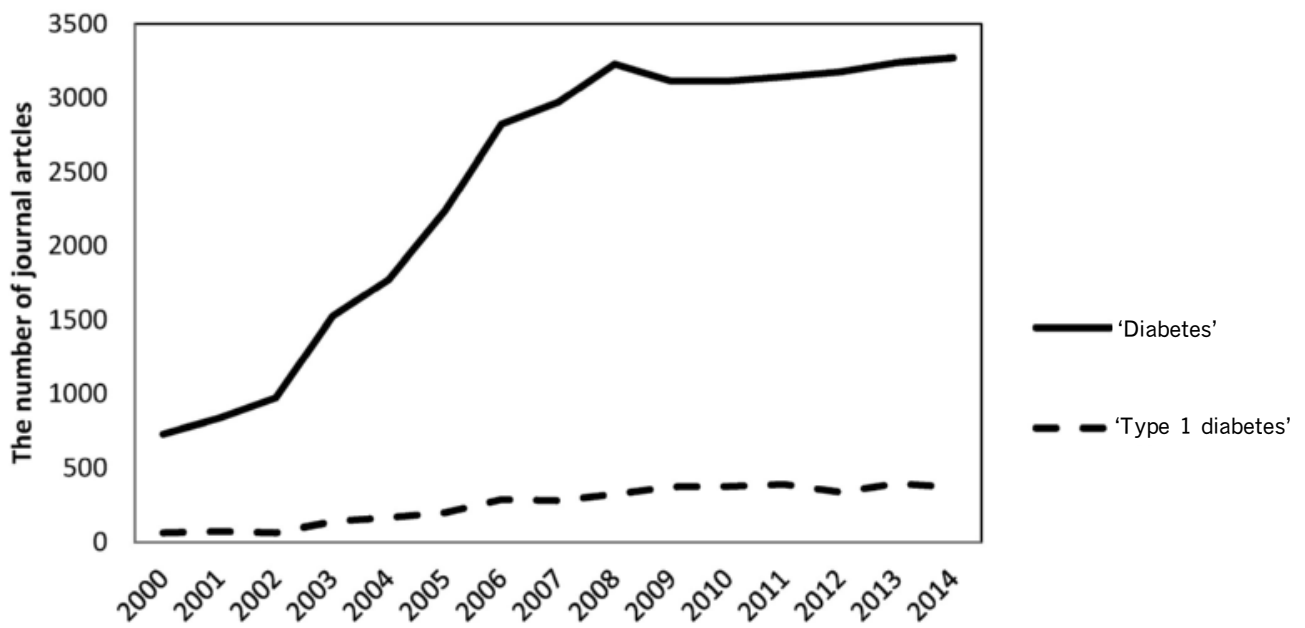

Fig. 2 Number of journal articles found in CINAHL/Hinari 2000 -2014 for the key words: 'diabetes' and 'Type 1 diabetes'.

School support, ${ }^{18}$ school based life experiences, ${ }^{19}$ self management problems in school, ${ }^{20}$ role of school nurse $^{21}$ school environment as factor releated to selfcare $^{17}$ were the main areas described under 'School behaviour'.

Family conflicts, ${ }^{22}$ family involvements, family management, ${ }^{23}$ family functioning, ${ }^{8}$ family emotions, ${ }^{24}$ self-efficacy and family cohesion, ${ }^{25}$ open family communication, ${ }^{26}$ family dysfunction, ${ }^{8}$ the role of family, ${ }^{27}$ family centred interventions, ${ }^{28}$ different family structures $^{29}$ were highlighted under 'family behaviour'.

\section{Discussion}

The results apparently denotes that the number of journal articles resulted in accordance with 'diabetes' and 'Type 1 diabetes' have increased gradually from 2000 to 2014. However the increasing trend is different because there were higher number of articles for 'diabetes' rather than 'Type 1 diabetes'. Both Type 1 and Type 2 diabetes have increased over the last century. Although the range of global variation on childhood T1DM was larger than before, the incidence of Type 2 diabetes is higher than the incidence of T1DM. ${ }^{3}$ Changes of human behavior and lifestyle have resulted 


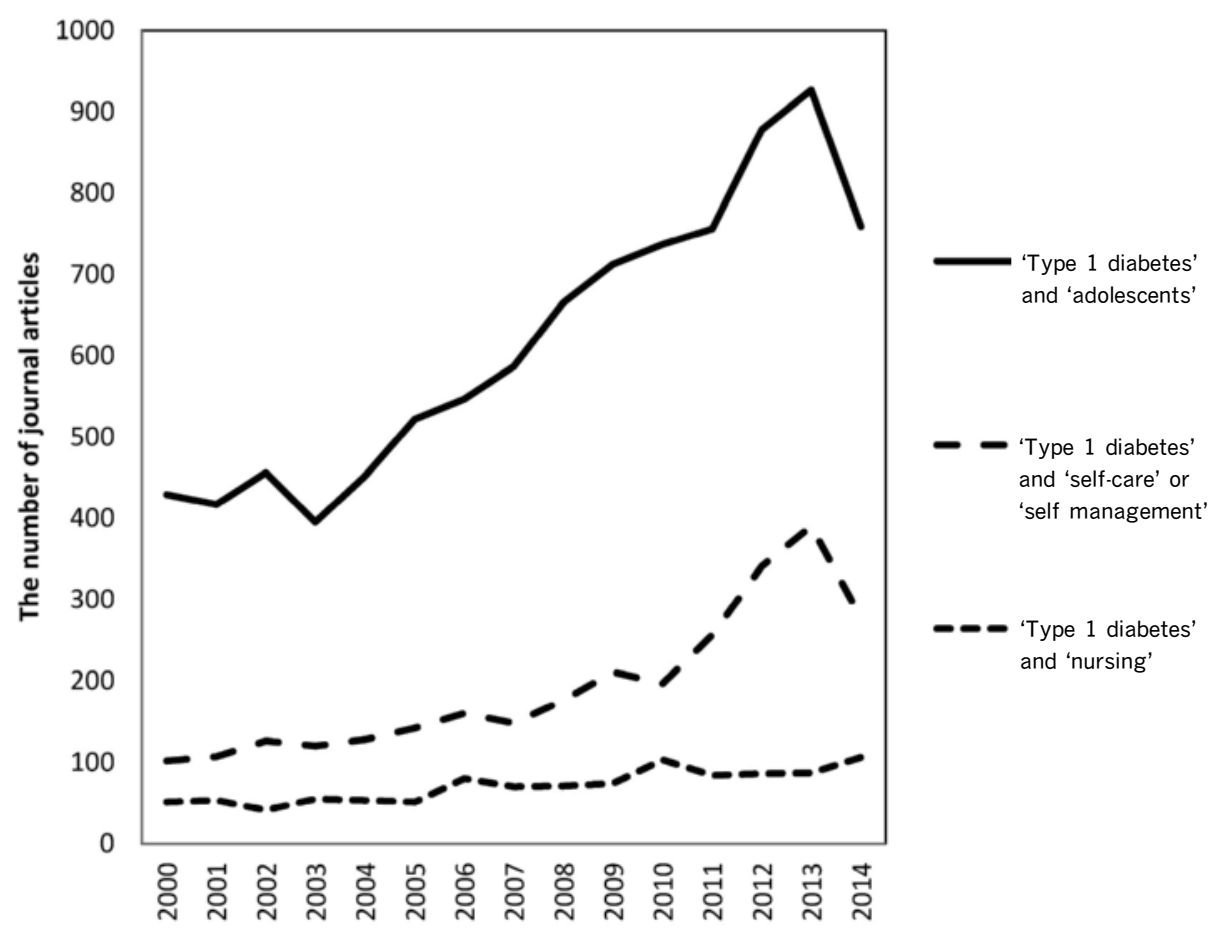

Fig. 3 Number of journal articles found in PubMed/Hinari 2000 -2014 for the multiple key words.

Fewer than 10 articles were found during all time periods for the key words 'Type 1 diabetes' and 'sociocultural' and 'Type 1 diabetes' and 'Sri Lanka'.

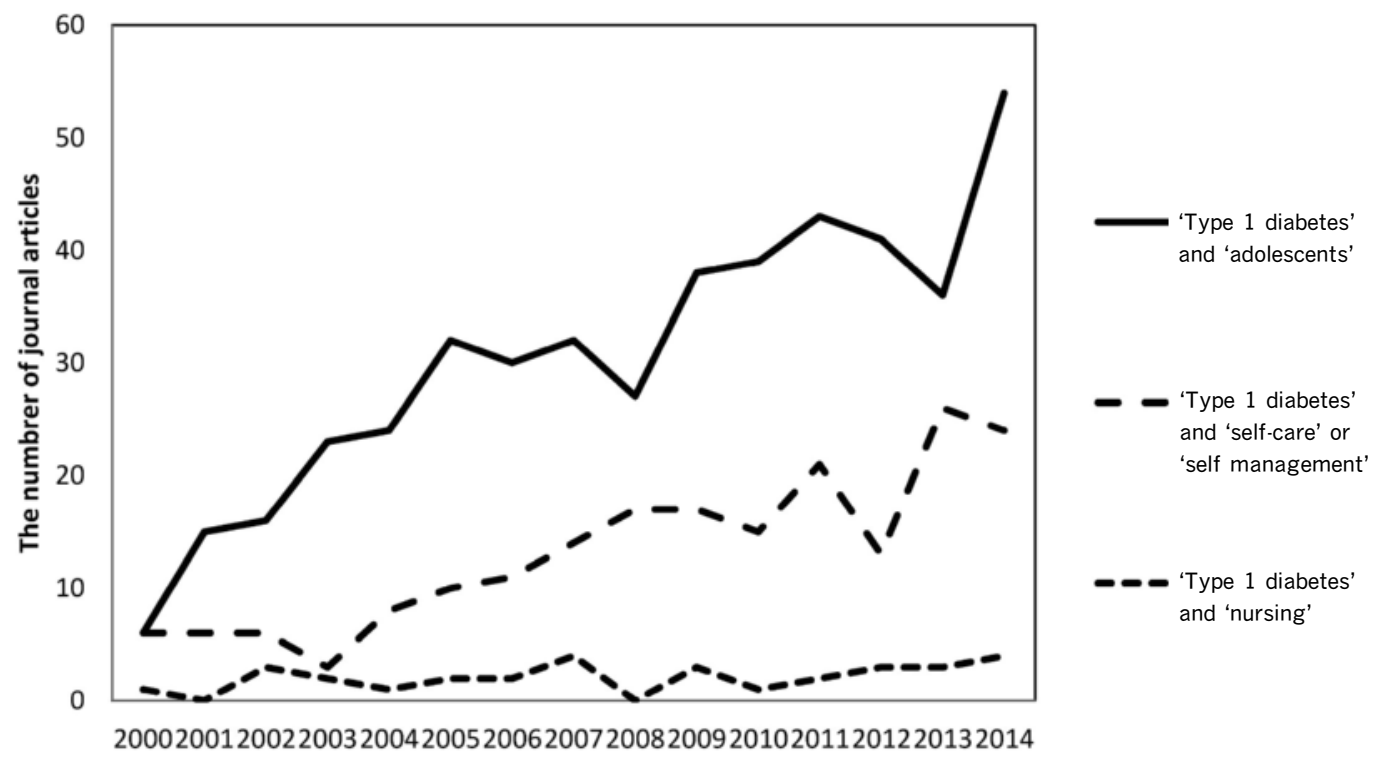

Fig. 4 Number of journal articles found in CINAHL/Hinari 2000-2014 for the multiple key words.

Fewer than 10 articles were found during all time periods for the key words 'Type 1 diabetes' and 'sociocultural' and 'Type 1 diabetes' and 'Sri Lanka'.

in a dramatic increase in the incidence of diabetes worldwide over the period. ${ }^{30}$ It has become a huge health problem worldwide. The increment of Type 2 diabetes and its long term effects can be controlled by the modifications made in life style and controlling personal and environment factors. As the literature highly oriented that area, the studies related to 'diabetes' were comparatively higher than the studies related to 'Type 1 diabetes'.

The systematic literature review emphasizes on the lack of studies related to 'Type 1 diabetes' within a specific sociocultural context. Behavioral modification is the frontline strategy to control this lifelong chronic illness. Cultural beliefs, values, and attitudes, confidence, habits, functional and cognitive abilities, support from others and the nature of health care 
access are some factors affecting self-care in diabetes management. ${ }^{31}$ Patients' cultural norms, attitudes, beliefs socio economic status and knowledge about disease affect the self-management of diabetes. ${ }^{32} \mathrm{Cul}$ turally appropriate behavioural modification should be followed in order to receive effective results. However there were hardly any articles on both 'Type 1 diabetes' and 'sociocultural' context in Sri Lanka.

An examination of parents' and adolescents' 'illness perceptions' and perceptions of diabetes management are crucial aspects to live with this chronic condition. The different perceptions between adolescents and parents result conflicts of diabetes management. The understanding of both adolescents' and parents' perceptions is vital. The experienced sociocultural background mostly connects with the perceptions of a person. Although many studies are available in the field in other sociocultural backgrounds in the world, the one and only pilot study is available in Sri Lankan sociocultural context. The results of the study indicate that there is a higher degree of positive illness perceptions among adolescents than parents, ${ }^{33}$ The study results similar to the results of the former studies. $^{7,34}$ The nature of cognition among adolescents according to their development stage might be the reason for the results. Greater understanding of illness perceptions between adolescents with T1DM and their parents is vital to facilitate sociocultural appropriate nursing care. The in-depth assessment of this field in Sri Lanka is beneficial in planning nursing care effectively.

The several findings in relation to 'associate characteristics' on self-care and metabolic control is a prominent area in the existing literature. Several studies revealed that there were different level of selfcare practices according to some characteristics such as age, gender, race, pubertal development and family factors. ${ }^{35,36}$ For example there was a deterioration in self-care practices as the children advance with age. ${ }^{12}$ Although the cognition and behavioural development are advance with age of children, the nature of their living environment could be negatively affected the self-care in disease management. The scientific studies, which reveal self-care practices among children with T1DM as advance with age, were not available in Sri Lanka. The highly competitive education system in Sri Lanka may be a major reason for deterioration of self-care practices among children and adolescents with T1DM in Sri Lanka. An association between gender and diabetes outcome were described in the majority of literature. The development pattern has differed as gender in children adolescents. Insulin resistant is a feature, which arises in pubertal period. As it is, vary of diabetes care outcome would occur within the gender difference. Ethnicity and family factors are mostly relate with sociocultural context. An examination of associated characteristics for self-care and metabolic control among adolescents with T1DM had not been adequately studied in Sri Lanka. Further scientific studies in this area, considering Sri Lankan sociocultural context is a vital aspect.

'School behaviour' is a major aspect in the literature. As stated in the literature, life experience in School is a dynamic learning process related to diabetes management. School aged children and adolescents experience close interaction with their peer group and school teachers. It is very important in performing the self-care practices in school. The children and adolescents with T1DM should exhibit more independent self-care practices and autonomy at school period rather than home. The school behaviour greatly affects with diabetes outcome. However there were hardly any scientific studies in this area in Sri Lanka. This aspect is considered to be a vital view point in future studies in Sri Lanka.

'Family behaviour' makes strong effects towards coping behaviour in the chronic condition, T1DM. The literature frequently discusses the self-care practices in relation to family functioning. Parents and members of family are the intimate people to children and adolescents in the Sri Lankan context. They greatly engage in diabetes management activities in children and adolescents. The process of sharing responsibilities in diabetes management from parents to children is seen in adolescence period. The scientific evaluation of this area is vital to organize nursing care plan for children and adolescents with T1DM.

The nature of family system is unique in Sri Lanka. The children and adolescents are closely bound with their parents and other family members within the Sri Lankan family system. The national survey on 'Emerging issues among adolescents in Sri Lanka' identified a strong influence between family and adolescents. School adolescents (10-14 years) were very close to their family $(60 \%) .32 \%$ of out of school adolescents (15-19 years) saw their family as caring and warm. $75 \%$ of adolescents thought they could depend on their families. ${ }^{37}$ The results of the study show a close relationship between Sri Lankan children and adolescents with their family members. The family is the crucial component of culture and thus the sharing of responsibilities in disease management among parents and adolescents would be a unique process within the Sri Lankan family system. The future studies in Sri Lanka should focus on this vital field for scientific assessment.

None of the available studies which discussed on how self-care and sharing responsibility in diabetes management is carried out among adolescents in Sri Lankan sociocultural context. Only two ethnographic studies were available related to 'diabetes' and 'Sri Lankan cultural context' in the literature. Both studies were Type 2 diabetes oriented. One explored health care behaviour of Sri Lankan Buddhist nuns ${ }^{38}$ and the other one studied diabetes health beliefs and practices among Sri Lankan adults. ${ }^{39}$

Nursing includes the promotion of heath, prevention of illness, and the care of ill, disabled and dying people. Advocacy, promotion of a safe environment, research, participation in shaping health policy and 
inpatient and health systems management, and education are also key nursing roles. ${ }^{40}$

Promoting an active, lifelong self-care process in disease management for the patient with the chronic disease is a fundamental function in nursing. Diabetes care education is the frontline strategy to assist patients in adapting a self-care management under this chronic condition. Nevertheless, the consideration made on this behalf within the Sri Lankan nursing education system is not adequate. Sri Lankan education system is developing diploma level to degree level at present. A very few nursing personals are engaging post graduate studies. The Post Basic College of Nursing is the recognized higher education institute which provides diploma level nursing education from $1960 .{ }^{41}$ It conducts post graduate diploma causes such as ward management, teaching and supervision, pediatric training, nursing in operating theatre etc, Yet it does not offer any special training on diabetes care or care of Non-Communicable Diseases (hereinafter NCDs). Almost all nursing officers are working in tertiary care units rather than primary health care sector in Sri Lanka. The public health nursing sisters involve in primary health care activities as middle level managers. Yet, community health nurses are not available. ${ }^{42}$ The public health midwife who involves in child and maternity care is the main community health worker in the community health sector in Sri Lanka at present. However midwives are not recognized to be involved in care of NCDs such as diabetes and assisting self-care practices among children and adolescents with T1DM. The continuing care and behavioural modification strategies are essential in prevention and controlling NCDs. The health care delivery system in Sri Lanka has not well designed to accommodate the goals of behavioural modification strategies. ${ }^{43}$ The special trained health care professionals for childhood diabetes care should be accessed the care of children with T1DM. It is recommended by American Diabetes Association that a nurse educated in the field of diabetes has a vital role in the diabetes care team. Unavailability of the active role of specialized nurse educator for childhood diabetes in Sri Lankan health care system is considered to be a draw back.

The management regimen of T1DM is complex and demanding. It requires both knowledge and skills. ${ }^{17}$ Self-care is the cornerstone to adapt into this condition. It is the key to a good physical and psychological outcome of the disease. ${ }^{44}$ Self-care is a daily and lifelong active process. It involves shifting and sharing responsibilities and decision making. ${ }^{45}$ This is specific within the context of adolescent development. Self-care process in diabetes management involves diabetes management activities such as: administering insulin, self-adjustment of insulin dose, monitoring blood glucose, regular diet planning exercise, updating knowledge of diabetes management and psychological adjustment. Self-care pattern may vary with the difference of sociocultural nature. Studies on populations from different cultures should be needed as ethnicity is a factor which influencesself management and metabolic control. ${ }^{20}$ The link between self-care sharing process and sociocultural diversity is not completely identified. ${ }^{46}$ The impact of sociocultural context on glycemic control behaviour is significant for adults with diabetes in Sri Lanka. ${ }^{39}$ The culturally appropriate interventions are recommended for promoting effective diabetes care. ${ }^{15}$ The existing literature emphasizes the importance of identify self-care behaviour among children and adolescents with T1DM in Sri Lanka.

The role of diabetes nurse educators cannot be neglected when assisting the self-care process in diabetes management. Upgrading nursing education in this specialized field and establishing nursing service in community health setup is highly recommended. The availability of a diabetic nurse educator who can be easily approached in both community and hospital setting is an important strategy to achieve goals of having quality self-care practices among children and adolescents with T1DM. Trained nurses available for 24 hours to assist patients and their families in Sri Lanka is recommended. ${ }^{4}$ Furthermore qualified and trained diabetes nurse educators should be utilized effectively within the Sri Lankan health care system for assisting self-care activities of children and adolescents with T1DM.

Specific scientific studies related to the process of self-care among Sri Lankan children and adolescents with T1DM are difficult to be found. An island wide standard method is not available to evaluate progress of the self-care practices among children and adolescents. It depends on several institutions which facilitate follow-up care for the adolescents with diabetes. A national guideline for the investigation and management of T1DM is recommended to overcome the challenges in the management of T1DM in children. ${ }^{4}$ The availability of well-organized sub specialized clinics for adolescents with diabetes may help to address specific problems encountered by them.

School support significantly and directly affected self-care behaviour and life satisfaction. ${ }^{18}$ The school based live experiences in Thaiwanese adolescents with T1DM showed multiple factors threatened at school health and well-being such as: identify the disease condition by others, social anxiety, ignorance from the classmates and transition to independent health management. ${ }^{19}$ School health nurses are not available in Sri Lanka. The school environment of children and adolescents with T1DM has not been scientifically studied by any researcher in Sri Lanka. The lack of school health programs and easily approachable regular diabetic health camps are some of the negative aspects in the management of the disease.

When taking the health care system specific to children and adolescents with T1DM in Sri Lanka into consideration, several issues can be identified. Issues of assisting self-care and sharing the process of selfcare, unavailability of childhood diabetes specific, updated nursing training, lack of accessible children 
and adolescent specific subspecialized clinics, lack of effective nursing interventions, unavailability of national guidelines for management of T1DM and insufficient involvement of scientific studies in the field of nursing care are noticeable.

Nurses in Sri Lanka should be engaged in key activities to achieve effective diabetes care outcomes among children and adolescents with T1DM. They should involve themselves in identifying the incidence of children and adolescents with T1DM, update knowledge and play an active role in ongoing education programs such as diabetes camp for children and adolescents. The education should be appropriate for the age, maturity, state of the disease condition, lifestyle, culture and family system of each patient. Furthermore the nurse should educate school teachers and assist in promoting self-care activities in schools. Moreover, implementing effective nursing interventions while taking sociocultural context into account and involved in scientific research studies on nursing care for children and adolescents with T1DM would help achieve effective diabetes care outcomes in Sri Lanka.

\section{Conclusion}

The nurses are responsible for empowering children and adolescents with T1DM by enhancing their knowledge and skills taking their development pattern and cultural appropriateness into consideration. It is the responsibility of the nurses to help the parents and family members to shift the responsibilities gradually to adolescents. Nursing interventions should consider specific self-care deficiencies identified within Sri Lankan sociocultural context. The scientific studies pertaining to this field and the nursing care for assisting self-care of children and adolescents need to be optimized in Sri Lanka.

\section{References}

1. Lissauer T, Clayden G. Illustrated text book of pediatrics, 2nd ed, Spain: Mosby International Limited. 2003: 337-344.

2. Marshall, M., Carter, B., Rose, K., et al. Living with type 1 diabetes: perceptions of children and their parents. J Clin Nursing 2009; 18(12): 1703-1710.

3. Karvonen M, Vink- Kajandra M, Moltchanava E, et al. Incidence of childhood type 1 diabetes worldwide. Diabetes Care 2000; 23: 1516-1526.

4. Atapattu N, De Silva KSH. Improving diabetes care in Sri Lankan children: the way forward. Sri Lanka J Diabetes Endocrinol Metabolism 2012; 2(1): 35-38.

5. Kesavadev J, Sadikot SM, Saboo B, et al. Challenges in Type 1 diabetes management in South East Asia: Descriptive situational assessment. Indian J Endocrinol Metabolism 2014; 18(5): 600-607.

6. Ramachandran A, Snehalatha C, Ma RCW. Diabetes in South-East Asia: an update. Diabetes Res Clin Practice 2014; 103(2): 231-237.

7. Amer KS. Children's views of their adaptation to type 1 diabetes mellitus. Pediatric Nursing 2008; 34(4): 281-288.
8. Leonard BJ, Garwick A, Adwan JZ. Adolescents' perceptions of parental roles and involvement in diabetes management. J Pediatric Nursing 2005; 20(6): 405-414.

9. Schmidt C. Mothers' perceptions of self-care in school-age children with diabetes. MCN. Am J Maternal Child Nursing 2003; 28(6): 362-370.

10. Schmidt C. Self-care in children with type 1 diabetes: a survey of mothers. MCN. Am J Maternal Child Nursing 2007; 32(4): 223-229.

11. Fisher KL. School nurses' perceptions of self-efficacy in providing diabetes care. J Sch Nursing 2006; 22(4): 223-228.

12. Dashiff CJ, McCaleb A, Cull V. Self-care of young adolescents with type 1 diabetes. J Pediatric Nursing 2006; 21(3): 222-232.

13. Neylon OM, O' Connell MA, Skinner TC, et al. Demographic and personal factors associated with metabolic control and self-care in youth with type 1 diabetes: a systematic review. Diabetes Metabolism Res Rev 2013; 29(4): 257-272.

14. Greene MS, Mandleco B, Roper SO, et al. Metabolic control, self-care behaviors, and parenting in adolescents with type 1 diabetes: a correlational study. Diabetes Educator 2010; 36(2): 326-336.

15. Guo J, Whittemore R, Grey $M$, et al. Diabetes selfmanagement, depressive symptoms, quality of life and metabolic control in youth with type 1 diabetes in China. J Clin Nursing 2013; 22: 69-79.

16. Dashiff C, Vance D, Abdullatif H, et al. Parenting, autonomy and self-care of adolescents with type 1 diabetes. Child Care Health Development 2009; 35(1): 79-88.

17. Kelo M, Martikainen M, Eriksson E. Self-care of school-age children with diabetes: an integrative review. J Adv Nursing 2011; 67(10): 2096-2108

18. Tang SM, Chen SW, Wang RH. Establishing a model to assess the effects of school support and self-care behaviours on life satisfaction in adolescents with type 1 diabetes in Taiwan. J Nursing Res 2013; 21(4): 244-251.

19. Wang YL, Brown SA, Horner SD. The school-based lived experiences of adolescents with type 1 diabetes. J Nursing Res 2013; 21(4): 235-243.

20. Hayes-Bohn R, Neumark-Sztainer D, Mellin A, et al. Adolescent and parent assessments of diabetes mellitus management at school. J Sch Health 2004; 74(5), 166-169.

21. Perry L, Steinbeck KS, Dunbabin JS, et al. Lost in transition? Access to and uptake of adult health services and outcomes for young people with type 1 diabetes in regional New South Wales. Med J Australia 2010; 193(8): 444-449.

22. Whittemore R, Liberti L, Jeon S, et al. Self-management as a mediator of family functioning and depressive symptoms with health outcomes in youth with type 1 diabetes. Western J Nursing Res 2014; 36(9): 1254-1271.

23. Beacham BL, Deatrick JA. Health care autonomy in children with chronic conditions: implications for self-care and family management. Nursing Clin North Am 2013; 48(2): 305-317.

24. Whittemore R, Jaser S, Guo J, et al. A conceptual model of childhood adaptation to type 1 diabetes. Nursing Outlook 2010; 58(5): 242-251.

25. Pinar R, Arslanoglu I, Isgüven P, et al. Self-efficacy and its interrelation with family environment and metabolic control in Turkish adolescents with type 1 diabetes. Pediatric Diabetes 2003; 4(4): 168-173.

26. Faulkner MS, Chang, LI. Family influence on self-care, quality of life, and metabolic control in school-age children and adolescents with type 1 diabetes. J Pediatric Nursing 2007; 22(1): 59-68.

27. Gunn KL, Seers K, Posner N, et al. "Somebody there to watch over you": the role of the family in everyday and 
emergency diabetes care. Health Social Care Commun 2012; 20(6): 591-598.

28. McBroom LA, Enriquez M. Review of family-centered interventions to enhance the health outcomes of children with type 1 diabetes. Diabetes Educator 2009; 35(3): 428438.

29. Dashiff C, Morrison S, Rowe J. Fathers of children and adolescents with diabetes: what do we know? J Pediatric Nursing 2008, 23(2), 101-119.

30. Zimmet P, Alberti KG, Shaw J. Global and societal implications of the diabetes epidemic. Nature 2001; 414(6865): 782-787.

31. Jaarsma RB, Strömberg A. A middle-range theory of selfcare of chronic illness. Adv Nursing 2012; 35 (3): 194-204.

32. Nam S, Chesla C, Stotts NA, et al. Barriers to diabetes management: patient and provider factors. Diabetic Res Clin Practice 2011; 93: 1-9.

33. Pathiranage AMSD, Mori Y, De Silva KSH. Illness perceptions among adolescents with type 1 diabetes mellitus and their parents. Proceedings of $3^{\text {rd }}$ Sri Lankan- Japan Collaborative Research-2015. Sri Lanka. 2015; p 29.

34. Herrman, JW. Children's and young adolescents' voices: perceptions of the costs and rewards of diabetes and its treatment. J Pediatric Nursing 2006; 21(3): 211-221.

35. Guo J, Whittemore R, He GP. The relationship between diabetes self-management and metabolic control in youth with type 1 diabetes: an integrative review. J Adv Nursing 2011; 67(11): 2294-2310.

36. Jaser SS, Faulkner MS, Whittemore R, et al. Coping, self-management, and adaptation in adolescents with type 1 diabetes. Ann Behavioral Med 2012; 43(3): 311-319.

37. Thalagala N, Rajapakse L. National survey on emerging issues among adolescents in Sri Lanka, Colombo. UNICEF
2004.

38. Idampitiya $\mathrm{C}$, Lenora $\mathrm{D}$, Lau $\mathrm{S}$, et al. Improving the quality of diabetes care in a Sri Lankan diabetes clinic through a diabetes nurse educator. Diabetes Res Clin Practice 2008; 80(1): 25-26.

39. Amarasekara AATD, Fongkaew W, Turale S, et al. An ethnographic study of diabetes health beliefs and practices in Sri Lankan adults. Int Nursing Rev 2014; 61(4): 507-514.

40. International Council of Nursing. Definition of nursing. Retrieved August 10, 2015, from http://www.icn.ch/whowe-are/icn-definition-of-nursing/

41. Pathiranage AMSD, Mori Y. The Japanese collaboration in nursing education in Sri Lanka: Proceedings of International Conference on Sri Lanka-Japan Collaborative Research-2014. Sri Lanka. 2014; p 2.

42. Jayasekara RS. Issues, challenges and vision for the future of the nursing profession in Sri Lanka: a review. Int Nursing Rev 2009; 56: 21-27.

43. Weeerasinghe $M$, Siriwardana D. Utilizing nurses as diabetes educators: Sri Lankan experience. 2013 (September 2014) http://ghf.g2hp.net/2013/09/16/utilizing-nurses-asdiabetic-educators-sri-lankan-experience/.

44. Schilling LS, Knafl KA, Grey M. Changing patterns of self-management in youth with type I diabetes. J Pediatric Nursing 2006; 21(6): 412-424.

45. Schilling LS, Grey M, Knafl KA. The concept of selfmanagement of type 1 diabetes in children and adolescents: an evolutionary concept analysis. J Adv Nursing 2002; 37(1): 87-99.

46. Helgeson VS, Siminerio L, Escoba O, et al. Predictors of metabolic control among adolescents with diabetes: A 4year longitudinal study. J Pediatric Psychol 2009; 34(3): 254-270. 